\title{
The Impact of Climate Change on Country's Fragility Assessment
}

\author{
Mengmeng $\mathrm{Li}^{1,2}$, Dongsheng $\mathrm{Bi}^{3}$, Dongfeng Yang ${ }^{4}$ \\ ${ }^{1}$ Key Laboratory of Land Environment and Disaster Monitoring, MNR, China University of Mining and Technology, Xuzhou, \\ China \\ ${ }^{2}$ School of Environment Science and Spatial Informatics, China University of Mining and Technology, Xuzhou, China \\ ${ }^{3}$ Department of Electronic Engineering, Fudan University, Shanghai, China \\ ${ }^{4}$ Department of Electronic Engineering, Northeast Petroleum University, Daqing, China \\ Email: 2257368793@qq.com
}

How to cite this paper: Li, M.M., Bi, D.S. and Yang, D.F. (2020) The Impact of Climate Change on Country's Fragility Assessment. Journal of Applied Mathematics and Physics, 8, 2447-2462.

https://doi.org/10.4236/jamp.2020.811181

Received: October 8, 2020

Accepted: November 21, 2020

Published: November 24, 2020

Copyright (c) 2020 by author(s) and Scientific Research Publishing Inc. This work is licensed under the Creative Commons Attribution International License (CC BY 4.0).

http://creativecommons.org/licenses/by/4.0/

\begin{abstract}
The damage costs of climate change have the potential to cause the breakdown of governmental structures. The paper focuses on how climate change impacts the state's fragility and how the government should intervene. The paper chooses temperature and rainfall as climate change indicators and establishes a comprehensive evaluating model. The weighting method of the model is determined by combining coefficient of variation method and improved entropy method. The model will output the objective comprehensive evaluation of state's fragility. The state's fragilities are quantitatively divided into three levels consisting of the fragile, the vulnerable and the stable in the model. For validation, this paper selects six representative countries and analyzes the degree and the approach of climate change's impacts on state's fragility. The fragile values by the model are consistent with the situation in these countries. The state's fragilities are also predicted by back propagation (BP) neural network. This paper analyzes the impacts of human interventions on improving state's fragility. The results indicate that the paper could provide reasonable suggestions for the government in the aspects of when and how to take what kind of interventions to improve state's fragility.
\end{abstract}

\section{Keywords}

State's Fragility, Climate Change, Comprehensive Evaluating Model, Objective Weighting Model

\section{Introduction}

The negative effects of climate change, including increased droughts, shrinking 
glaciers, changing animal and plant ranges and sea-level rise, vary from region to region and they are already being realized. Many of these effects will alter the way people live and may have the potential to cause the weakening and breakdown of social and governmental structures. Moreover, destabilized governments could result in fragile states. A fragile state is the one where the state government is not able to, or chooses not to provide the basic essentials to its people. Being a fragile state increases the vulnerability of a country's population suffering from the impact of such climate shocks as natural disasters, decreasing arable land, unpredictable weather, and increasing temperatures.

Many scholars at home and abroad began to pay attention to the role of climate change in boosting and impeding the vulnerability of the country. In the 1970s and 1990s, Adger W N [1] analyzed the social vulnerability based on the volatile and extreme climate of coastal cities; $\mathrm{Yu} \mathrm{Ou} \mathrm{[2]} \mathrm{applied} \mathrm{model} \mathrm{simula-}$ tions, the single index method and the comprehensive index method to quantitatively summarize the vulnerability of regional climate change and promote research and development in this area. Xue He [3] used the Analytic Hierarchy Process to establish a fragility evaluation index system, which applied the Likert five-point method to predict the degree of national stability and analyze the country's Fragility index. Zhendong Peng [4] put forward the evaluating prediction model to determine the fragility of a state and the influence of climate change. By applying improved model intervention, Kewei Chen [5] established a model to measure a country's fragility and the impact of climate changes on fragility. Plans were put forward to mitigate the risks posed to a country by climate change. Qilan Chen [6] established the evaluation system model of fragility based on the Analytic Hierarchy Process (AHP) to analyze how climate change directly or indirectly affects fragility.

However, the above papers didn't systematically analyze the impact of climate change on state fragility. It was found a lack of analysis about which state-driven interventions could mitigate the risk of climate change and how to prevent a country from becoming a fragile state. Therefore, the paper firstly establishes an appropriate evaluating model to evaluate the fragility of a country and measures the influences of climate change simultaneously. Furthermore, the model is applied to specific countries, and the paper measures how climate change impacts the country's fragility and predicts the national fragility. The impact of human interventions to improve a state's fragility on the total national GDP is also analyzed in this study. The nomenclatures used in this study are provided in Table 1 to help the reader conveniently follow the paper.

\section{State Fragility Comprehensive Evaluation Model}

\subsection{Construction of Evaluation Index System}

To assess the state's fragility, the paper firstly divides the assessment system into four dimensions: social, economic, political, cohesion, and selects twelve representative indicators to construct the comprehensive evaluating index system of 
Table 1. Symbols used in this study and the corresponding descriptions.

\begin{tabular}{|c|c|}
\hline Symbols & The descriptions of symbols \\
\hline$\sigma_{i}$ & the standard deviation of the $i$ indicator \\
\hline $\bar{X}_{i j}$ & the average of the $i$ indicator \\
\hline$\delta_{i}$ & the coefficient of variation of the $i$ indicator \\
\hline$P_{i j}$ & the normalized value obtained after the coordinate translation method \\
\hline$Z$ & coordinate panning magnitude \\
\hline$w_{i}$ & combination weights \\
\hline$R_{\text {En }}$ & the coefficient of difference degree \\
\hline$w_{j}$ & the weight of rainfall to fragility of the country \\
\hline$w_{w}$ & the weight of temperature to fragility of the country \\
\hline$w_{i}^{\prime}$ & improved weight of twelve fragile states index \\
\hline$R_{i j}$ & the proportion of indicators \\
\hline$e_{i}$ & the entropy value of the $i$ indicator \\
\hline$g_{i}$ & the difference coefficient of the $i$ indicator \\
\hline$w_{1 i}$ & the weight of variation of the $i$ indicator \\
\hline$w_{2 i}$ & the weight of improved entropy method of the $i$ indicator \\
\hline$p_{1}, p_{2}, \cdots, p_{n}$ & the weight values of each evaluation indicators which are arranged in descending order \\
\hline$x_{1 i}$ & the correlation coefficient of the rainfall to the $i$ indicator of state's fragility \\
\hline$x_{2 i}$ & the correlation coefficient of the temperature to the $i$ indicator of state's fragility \\
\hline$s_{1 i}$ & $\begin{array}{l}\text { the normalized data of the correlation coefficient of the rainfall to the } i \text { indicator of } \\
\text { state's fragility }\end{array}$ \\
\hline$S_{2 i}$ & $\begin{array}{l}\text { the normalized data of the correlation coefficient of the temperature to the } i \\
\text { indicator of state's fragility }\end{array}$ \\
\hline$\Delta w_{1 i}$ & the correction value of weight of rainfall to the $i$ indicator of state's fragility \\
\hline$\Delta w_{2 i}$ & the correction value of weight of temperature to the $i$ indicator of state's fragility \\
\hline$w_{i}^{\prime}$ & corrected weights \\
\hline$W_{i j}$ & initial weight between Hidden layer and Input layer \\
\hline$x_{i}(p)$ & Input of neural network \\
\hline$a_{j}(p)$ & the actual output of hidden layer \\
\hline$v_{j r}$ & the weights between the output and hidden layers \\
\hline
\end{tabular}

state's fragility.

Second, the thesis chooses temperature and rainfall as indicators of climate change that can indirectly increase fragility as it influences other factors and indicators. See Figure 1 for details. 


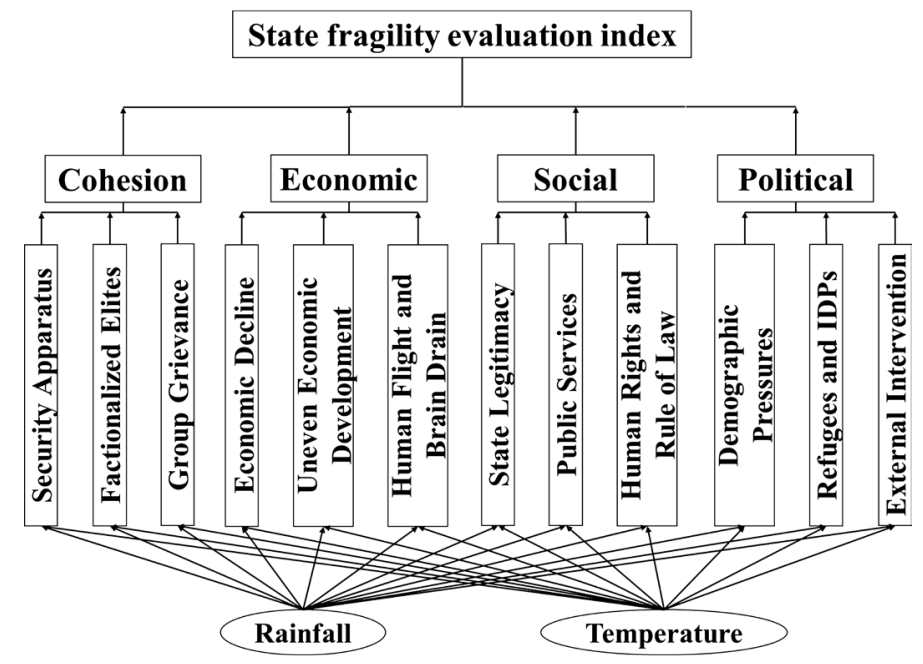

Figure 1. Indicators affecting state's fragility.

\subsection{Weighting Model of Indexes on State's Fragility}

To quantitatively characterize the fragility of a country, the paper calculates the weight of twelve indexes on state's fragility. Considering the objectivity of determining weight, the thesis chooses the method that weight will be determined by objective combination to figure out the weights, making the comprehensive evaluating system more scientific and reasonable [7]. The methods to determine weights include the coefficient of variation method, the improved entropy method, and the combination method.

\subsubsection{Coefficient of Variation Method}

The coefficient of variation method directly uses the information of each evaluating indicators to empower each indicator. The method empowers by referring to the degree of variation of the original measurement value of each index among all the evaluating indicators. If the coefficient of variation of evaluating indicator is larger, it will have a more significant result in the whole index system, so it is also given a larger weight. On the contrary, if the coefficient of variation of the indicator is small, it will produce less effect and will be given a smaller weight. This method can effectively overcome the shortcomings of subjective empowerment to more objectively reflect the relationship between indicators. The specific calculation process of the coefficient of variation is as follows: (There are $n$ evaluating indicators, each indicator has $\mathrm{m}$ years)

1) The standard deviation of each indicator is calculated as:

$$
\sigma_{i}=\sqrt{\frac{\sum_{j=1}^{m}\left(x_{i j}-\bar{x}_{i j}\right)^{2}}{m}}
$$

where: $\sigma_{i}$ is the standard deviation of $i$ indicator; $\bar{X}_{i j}$ is the average of $i$ indicator.

2) The coefficient of variation of each indicator is calculated as:

$$
\delta_{i}=\sigma_{i} / \bar{x}_{i j}
$$


where: $\delta_{i}$ is the coefficient of variation of $i$ indicator.

3) The weight of each evaluating indicators is calculated as:

$$
w_{1 i}=\delta_{i} / \sum_{i=1}^{n} \delta_{i}
$$

where: $w_{1 i}$ is the weight of variation of $i$ indicator

\subsubsection{Improved Entropy Method}

In information theory, entropy is a measure of uncertainty. The improved entropy method determines the weight of the indicator by the amount of information transmitted by each indicator received by policymakers. In general, the entropy of information reflected by an indicator is the smaller, the amount of information transmitted by the indicator is the more, and it can play a more significant role in the comprehensive evaluation. Therefore, the weight corresponding to the indicator is also more considerable. If the information entropy of an indicator is more remarkable, its corresponding weight is smaller. Specific steps of calculating are as follows:

1) Make indexes being dimensionless

As the dimension and magnitude of the evaluating indicator are inconsistent, to eliminate the influence caused by the above factors, the process needs to standardize the data (use $Z$-score method) before using the entropy method to determine the weight. The formula on standardization of indicator is:

$$
x_{i j}^{\prime}=\frac{x_{i j}-\bar{x}_{i j}}{\sigma_{i}}
$$

The normalized values have positive and negative values, and the process uses the coordinate translation method to eliminate the influence of negative values on the subsequent calculation. The method is as follows:

$$
P_{i j}=x_{i j}^{\prime}+Z
$$

where: $P_{i j}$ is the normalized value obtained after the coordinate translation method; $Z$ is coordinate panning magnitude, and its value can be determined by the minimum value of $x_{i j}^{\prime}$. Under normal circumstances, $Z$ is an integer, the range is $[0,5]$. The closer the value of $Z$ is to 0 , the better the credibility of the evaluating results.

2) Determine the proportion of indicators

$$
R_{i j}=P_{i j} / \sum_{j=1}^{m} P_{i j}
$$

3) Calculate the entropy power $e_{i}$ of $i$ indicator

$$
e_{i}=-\frac{1}{\ln m} \sum_{j=1}^{m} R_{i j} \cdot \ln R_{i j}
$$

4) Calculate the difference coefficient $g_{i}$ of $i$ indicator

$$
g_{i}=1-e_{i}
$$

5) Calculate the weight $w_{2 i}$ of the $i$ indicator 


$$
w_{2 i}=g_{i} / \sum_{i=1}^{n} g_{i}
$$

\subsubsection{The Combination Method to Determine the Weight}

The method of objective empowerment can rule out the influence of subjective factors. However, due to the limitation of the calculation method, the result of weighting on the same indicator system may be greatly different. To minimize the limitations of various objective weighting method and the differences of calculation results, and to improve the scientific evaluation, the method combines the two kinds of objective empowerment method to get the weight of indicator. The formula used is:

$$
w_{i}=(1-a) w_{1 i}+a w_{2 i}
$$

where: $w_{i}$ is the weight of combination; $w_{1 i}$ and $w_{2 i}$ are the weight of evaluating indicator calculated by the coefficient of variation method and the improved entropy method. The value of $a$ is determined by the coefficient of variation method and calculated by the following formula. The formula for calculating is as follows:

$$
a=R_{E n} \cdot \frac{n}{n-1}
$$

where: $R_{E n}$ is the coefficient of different degree. When the weight and the role of each indicator are equal, the difference coefficient obtained is 0 . When the weight and the role of each indicator have a larger difference, the coefficient of different degree is also larger. According to the principle of the degree of difference, the coefficient of different degree can be calculated according to the following formula:

$$
R_{E n}=\frac{2}{n}\left(1 \cdot p_{1}+2 \cdot p_{2}+\cdots+n \cdot p_{n}\right)-\frac{n+1}{n}
$$

where: $n$ is the number of indicators; $p_{1}, p_{2}, \cdots, p_{n}$ is the weight of each evaluating indicator that is arranged in descending order.

It can be seen that when the weight of each evaluating indicators obtained by the coefficient of variation method is the same, and the size is $1 / n$, the coefficient of difference degree takes the minimum value 0 . When the weight of each indicator is much different, it can be assumed that there will be only one indicator that is much larger than the other indicators. And its weight value can be approximately considered 1 , the coefficient of variation close to $n-1 / n$, and $a \approx 1$. Therefore, the paper can infer that the value of $R_{E n}$ is $[0,1)$.

\subsection{Improve Weights of the Index on State's Fragility}

Climate change has the relevance to each index of state's fragility in a certain degree. So as to improve the accuracy of the model, based on the correlation coefficient between two climate change indicators and each index of state's fragility, the paper divides the weight of two climate change indicators on the state's fragility into twelve indexes of national fragility in proportional. 
In the weight distribution model, the paper firstly normalizes the correlation coefficients of rainfall and temperature to twelve indexes of the country's fragility, respectively.

$$
\begin{aligned}
& S_{1 i}=\frac{x_{1 i}}{\sum_{i=1}^{12} x_{1 i}} \\
& S_{2 i}=\frac{x_{2 i}}{\sum_{i=1}^{12} x_{2 i}}
\end{aligned}
$$

where: $x_{1 i}$ is the correlation coefficient of rainfall to $i$ index of state's fragility; $x_{2 i}$ is the correlation coefficient of the temperature to $i$ index of state's fragility; $s_{1 i}$ is the normalized data of the correlation coefficient of the rainfall to $i$ index of state's fragility; $s_{2 i}$ is the normalized data of the correlation coefficient of the temperature to $i$ index of state's fragility.

Secondly, the normalized values are respectively multiplied by the weights of rainfall and temperature to obtain the correction value of weight.

$$
\begin{aligned}
& \Delta w_{1 i}=s_{1 i} \cdot w_{j} \\
& \Delta w_{2 i}=s_{1 i} \cdot w_{w}
\end{aligned}
$$

where: $\Delta w_{1 i}$ is the correction value of the weight of rainfall to $i$ index of state's fragility; $\Delta w_{2 i}$ is the correction value of the weight of temperature to $i$ index of state's fragility; $w_{j}$ is the weight of rainfall to country's fragility; $w_{w}$ is the weight of temperature to national fragility.

Finally, the correction value of weight is added to the corresponding weight to obtain the corrected weight.

$$
w_{i}^{\prime}=w_{i}+\Delta w_{1 i}+\Delta w_{2 i}
$$

where: $w_{i}^{\prime}$ is the corrected weight of twelve indexes of country's fragility; $w_{i}$ is the uncorrected weight of twelve indicators of state's fragility.

\subsection{Determine and Classify the Comprehensive Evaluating Result of State's Fragility}

To characterize the country's fragility, it is also necessary to standardize the value of twelve indexes on the state's fragility to evaluating indicators. The value of each index is the larger, corresponding country's fragility is the greater. Therefore, the data is equivalent to the evaluating index. The range of each evaluating index is $(0,10]$, which is equal to the already standardized data. The paper chooses the value of indexes of each state's fragility as the evaluating indicator. The uncorrected weights of the twelve indexes of state's fragility are multiplied by the value of indexes on each country's fragility to obtain the results of the comprehensive evaluating of the state's fragility. Because the range of index for each state's fragility is $(0,10]$, the range of the comprehensive evaluating results of the state's fragility is $(0,10]$. The paper uses the comprehensive evaluating results to characterize the state's fragility [8].

In order to identify state's fragility, the state's fragility is classified as fragile, 
vulnerable, and stable. Level of the state's fragility is shown in Table 2.

The paper applied the model to Sudan, Egypt, China, Brazil, Greece and Finland to calculate the comprehensive evaluating results of six countries' fragility in the last ten years. Comprehensive evaluating results of the fragility are shown in Figure 2.

By the comparison between the comprehensive evaluating results and the actual situations, the paper can get a conclusion that the results are consistent and the model is feasible and reasonable.

\section{The Way on Climate Change Impact Fragility of Sudan and Greece}

\subsection{Impacts of Climate Change on Fragility of Sudan and Greece}

As Sudan is one of the top ten most fragile countries, and Greece isn't among the ten most fragile states, Sudan and Greece are chosen as the research target. Firstly, the paper figures out the fragility of Sudan and Greece with and without the influence of climate change in the last decade by using the comprehensive evaluating model to study the impact of climate change on the fragility of Sudan and Greece. Fragility is shown in Figure 3.

The comparative analysis shows that climate change has significantly increased the fragility of Sudan and has brought Sudan from a vulnerable country to a fragile country. On the contrary, Greece is still in the vulnerable stage under

Table 2. Level of the state's fragility.

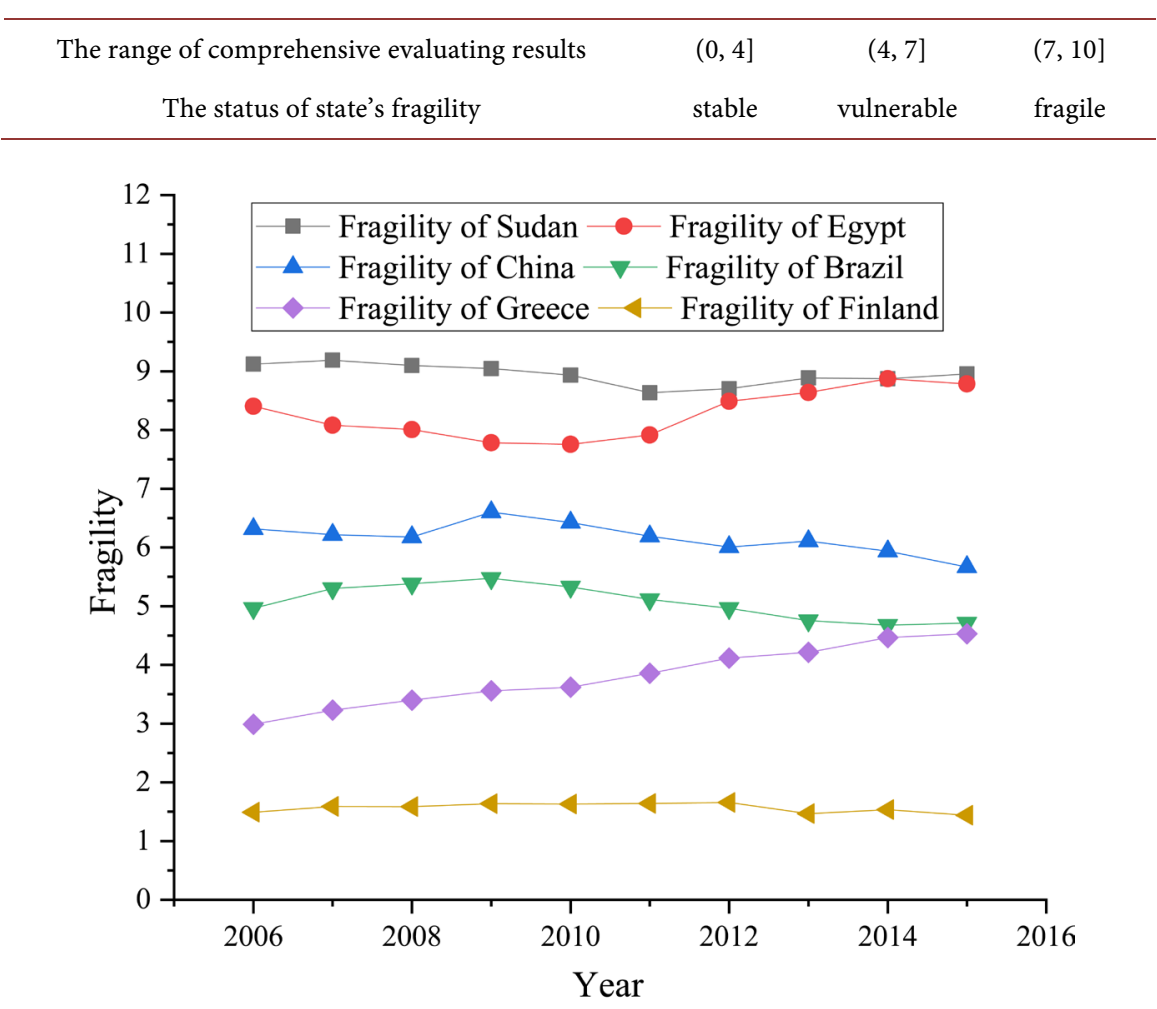

Figure 2. Comprehensive evaluation of the fragilities in six representative counties. 


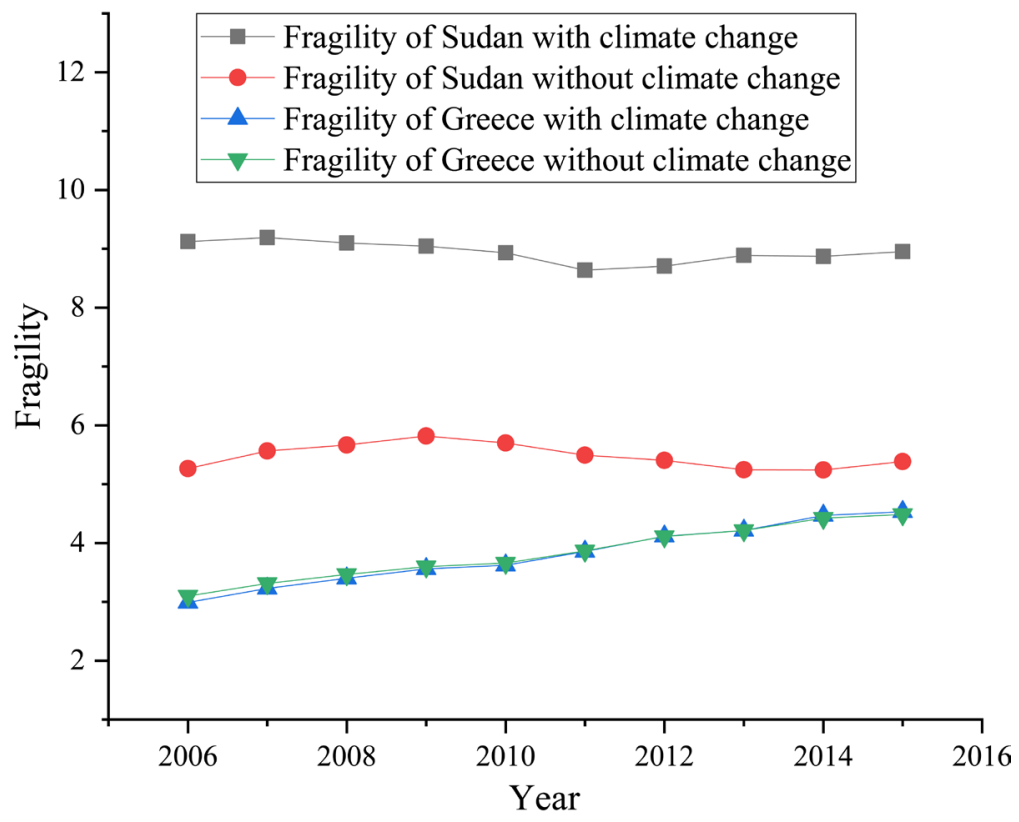

Figure 3. The fragility of Sudan and Greece with and without climate change.

the influence of climate change, but climate change has slightly increased the fragility of Greece in the past few years.

\subsection{How Climate Change Affects Fragility of Sudan and Greece}

The paper conducted a comparative analysis of the change in weight and correlation with and without climate change to explore the ways by which climate change affects the fragility of Sudan and Greece, and used the objective combination weighting method to figure out the weight of indexes on Sudan and Greece fragility with and without the influence of climate change [9]. As shown in Table 3.

The results show that: Climate change affects the fragility of Sudan mainly by affecting the weight of Security Apparatus and Economy, as well as affect the fragility of Greece primarily by impacting the weight of Human Flight, Brain Drain, Demographic Pressures.

Then, SPSS distance correlation analysis is used to find the Pearson correlation coefficient among two climate change indicators and twelve indexes of Sudan and Greece fragility, which is shown in Table 4.

The results show that rainfall and temperature of Sudan have the largest positive correlation with Security Apparatus and Demographic Pressures of Sudan respectively, as well as the most considerable negative correlation with Refugees and IDPs of Sudan. Therefore, they indirectly affect the fragility of Sudan mainly by promoting the Security Apparatus, Demographic Pressures, and suppressing Refugees and IDPs of Sudan. Meanwhile, rainfall and temperature of Greece, respectively, has the largest positive correlation with State Legitimacy and Security Apparatus of Greece, as well as the most considerable negative correlation with Demographic Pressures, Human Flight and Brain Drain, thus they indirectly 
Table 3. The weight of index on Sudan and Greece fragility with and without Climate Change.

\begin{tabular}{ccccc}
\hline \multirow{2}{*}{ states fragility index } & \multicolumn{3}{c}{ Weight with climate change } & \multicolumn{2}{c}{ Weight without climate change } \\
\cline { 2 - 5 } & Sudan & Greece & Sudan & Greece \\
\hline Security Apparatus & 0.065768 & 0.10739 & 0.032518 & 0.097678 \\
Factionalized Elites & 0.042564 & 0.15779 & 0.071233 & 0.15548 \\
Group Grievance & 0.036424 & 0.073954 & 0.0302 & 0.062916 \\
Economy & 0.18326 & 0.12999 & 0.21399 & 0.12615 \\
Economic Inequality & 0.12967 & 0.025945 & 0.15393 & 0.040314 \\
Human Flight and Brain Drain & 0.094324 & 0.038218 & 0.087751 & 0.056947 \\
State Legitimacy & 0.078795 & 0.098605 & 0.064483 & 0.091997 \\
Public Services & 0.1054 & 0.061218 & 0.098526 & 0.050381 \\
Human Rights & 0.07885 & 0.056603 & 0.066483 & 0.049276 \\
Demographic Pressures & 0.11565 & 0.042074 & 0.099031 & 0.060706 \\
Refugees and IDPs & 0.0086132 & 0.10629 & 0.037659 & 0.10735 \\
External Intervention & 0.060687 & 0.10192 & 0.044195 & 0.10081 \\
\hline
\end{tabular}

Table 4. Pearson correlation coefficients among rainfall, temperature and states fragility indexes.

\begin{tabular}{ccccc}
\hline \multirow{2}{*}{ States fragility indexes } & \multicolumn{2}{c}{ Correlation with rainfall } & \multicolumn{2}{c}{ Correlation with temperature } \\
\cline { 2 - 5 } & Sudan & Greece & Sudan & Greece \\
\hline Security Apparatus & 0.582 & 0.264 & 0.554 & 0.495 \\
Factionalized Elites & 0.116 & 0.36 & -0.257 & 0.339 \\
Group Grievance & 0.246 & 0.306 & 0.163 & 0.428 \\
Economy & 0.186 & 0.247 & 0.191 & 0.368 \\
Economic Inequality & -0.066 & -0.317 & 0.15 & -0.221 \\
Human Flight and Brain Drain & 0.105 & -0.301 & 0.404 & -0.336 \\
State Legitimacy & 0.165 & 0.421 & 0.44 & 0.295 \\
Public Services & 0.148 & 0.11 & 0.437 & 0.479 \\
Human Rights & 0.093 & 0.197 & 0.431 & 0.313 \\
Demographic Pressures & 0.262 & -0.411 & 0.578 & -0.272 \\
Refugees and IDPs & -0.102 & -0.109 & -0.333 & 0.315 \\
External Intervention & 0.033 & 0.227 & 0.437 & 0.217 \\
\hline
\end{tabular}

impact the fragility of Greece mainly by promoting the State Legitimacy and Security Apparatus, as well as suppressing Demographic Pressures, Human Flight and Brain Drain of Greece.

In summary, climate change significantly increases the fragility of Sudan and transforms into fragile from vulnerable by affecting Security Apparatus, Demographic Pressures, Refugees and IDPs indexes. Consider Sudan's economy and 
geographical location and other factors (i.e., Sudan is a developing country and near the Sahara Desert), thus climate change has a great effect on indexes of Sudan's fragility and makes the fragility of Sudan significantly increase in the short term. Differently, climate change increases the fragility of Greece through four definitive indicators: State Legitimacy, Demographic Pressures, Security Apparatus, Human Flight and Brain Drain, and climate change affect the fragility of Greece to a small extent.

\section{BP Neural Network Model of the Fragile Tipping Point}

\subsection{BP Neural Network Prediction Model}

Back propagation (BP) neural network is a kind of neural network with three or more layers of neurons, including input layer, intermediate layer and output layer. The upper and lower layers are fully connected, but there is no connection between the same layer of neurons. There are network weights between the input neuron and the hidden layer neuron, which means the connection strength between the two neurons. Any neuron in the hidden layer or output layer integrates the information from all the neurons in the previous layer, and multiplies by a threshold to the integrated information. This mainly imitates the principle that neurons in biology must reach a certain threshold before being triggered, and then the integrated information will be used as the input of neurons. When a pair of learning samples are provided to the input neurons, the activation value of neurons (the output value of neurons in this layer) propagates from the input layer through each hidden layer to the output layer. Each neuron in the output layer obtains the input response of the network. According to the direction of reducing the error between the output of the network and the actual output samples, it reversely passes through each hidden layer and returns to the input layer from the output layer to modify the connection weights step by step.

The BP neural network model in our study was illustrated as Figure 4. The network inputs are fragilities of countries from 2006 to 2015 and the network

\section{BP neural network model}

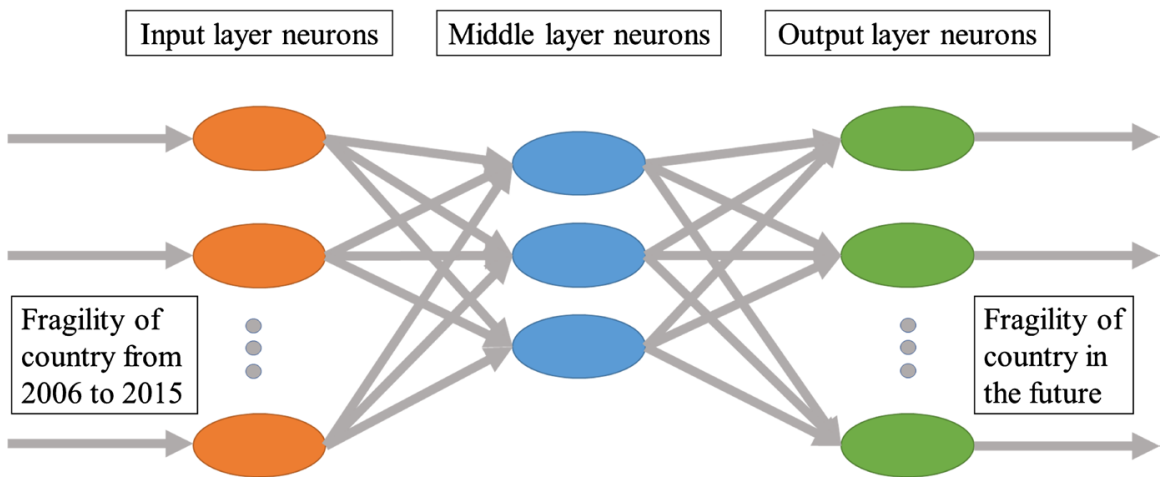

Figure 4. BP neural network model applied in our study to predict the fragility in the future based on the fragilities in the past ten years. 
output is the predicted fragility.

1) Randomly give initial weight $W_{i j}$ among Hidden layer and Input layer.

2) Enter $x_{i}(p)$ from the given sample. Calculate the actual output of hidden layer $a_{j}(p)$. Then write the threshold in Neural Networks to the connection right. Make hidden threshold $\theta_{j}=W_{n j}, x_{n}=-1$, then:

$$
a_{j}(p)=f\left(w_{i j} x_{i}(9)\right)(j=1,2, \cdots, m-1)
$$

3) Calculate the weight between the output and hidden layers $v_{j r}$ Let the neurons in the output layer as the target, and take the given output target $\operatorname{tr}(p)$ as the polynomial value of the equation. Then establish equations, expressed as a linear system of equations:

$$
\left\{\begin{array}{c}
a_{0}(1) v_{1 r}+a_{0}(1) v_{1 r}+\cdots+a_{m}(1) v_{m r}=\operatorname{tr}(1) \\
a_{0}(2) v_{1 r}+a_{0}(2) v_{1 r}+\cdots+a_{m}(2) v_{m r}=\operatorname{tr}(2) \\
\vdots \\
a_{0}(p) v_{1 r}+a_{0}(p) v_{1 r}+\cdots+a_{m}(p) v_{m r}=\operatorname{tr}(p)
\end{array}\right.
$$

Abbreviated as $A v=T$.

In order to make the system have a unique solution, matrix $A$ of the equation is a non-singular matrix. Its rank is equal to the rank of its augmented matrix, which is: $r(A)=r(A \mid B)$, and the number of equations equals the number of unknowns, so $m=p$. At this time, the only solution to the system of equations is:

$$
V_{r}=\left[v_{0 r}, v_{1 r}, \cdots, v_{m r}\right](r=0,1,2, \cdots, m-1)
$$

4) Repeat the third step to find out the output layer m neurons weight, the weight matrix of the output layer plus the randomly fixed hidden layer, and input layer weights is equal to the last trained weight matrix of the neural network.

\subsection{BP Neural Network Prediction Results}

Through BP neural network prediction model, the paper predicts fragility in Greece in the next 200 years. What's more, the figure of fitting results of the BP neural network is drawn, and the paper compares the predicted value with the real value. The fitting results of the BP neural network model are shown in Figure 5.

It can be seen the fitting result of the BP neural network is excellent. Moreover, the prediction results show that Greece will reach the tipping point in 2167, and the fragility will reach 7 , which means after 147 years, Greece will transform from a vulnerable country to a fragile state. Therefore, it will be of great value to apply the BP neural network to a wider range of countries to further confirm its efficacy in the future exploration.

Taking the national strength into consideration, Greece is a developed country and closed to the Mediterranean. Therefore, the impact of climate change on indexes of state's fragility, such as economic indicators, is small. Considering the effects of a worsening climate such as increased droughts, shrinking glaciers, changes in the range of flora and fauna, rising sea levels, Greece will reach a very 


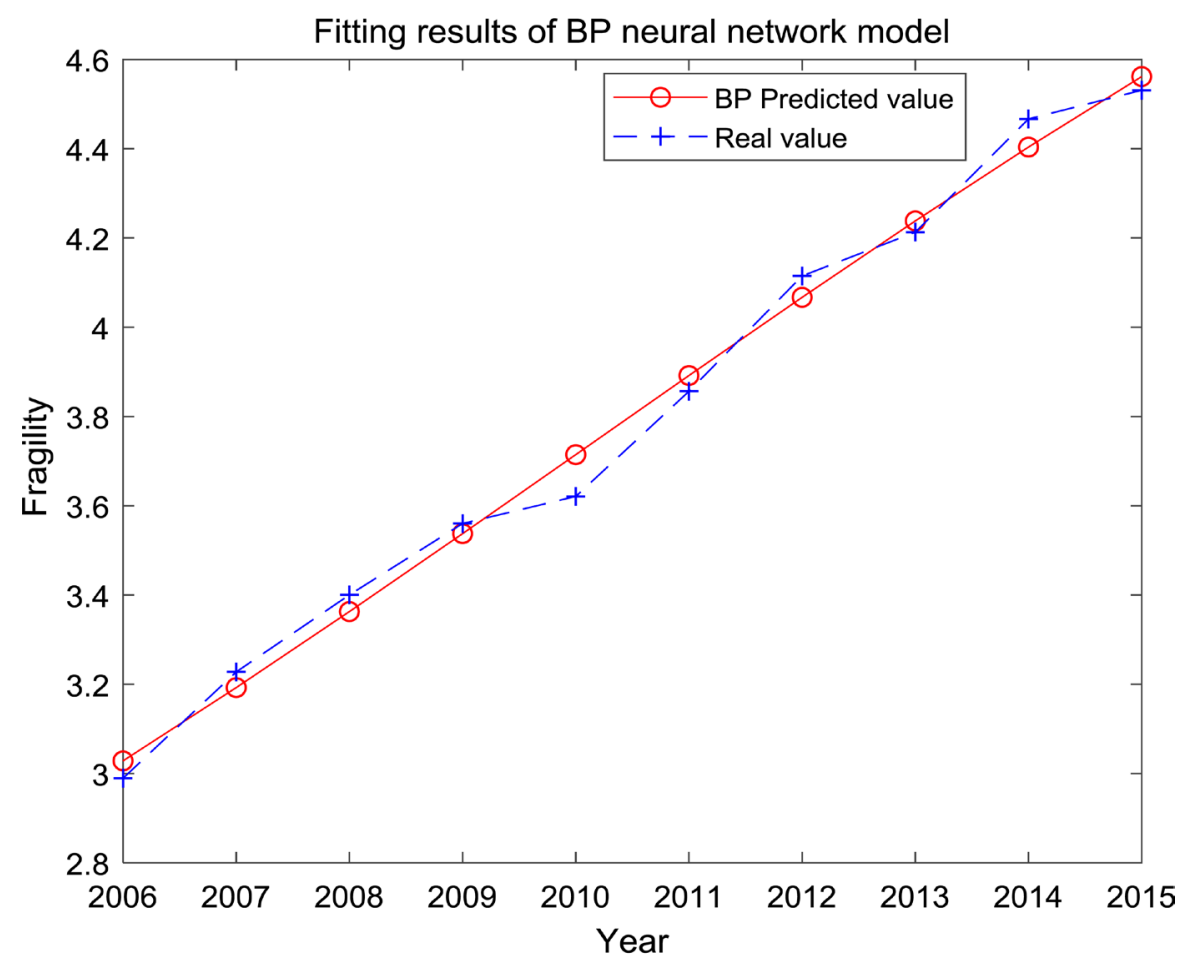

Figure 5. Fitting results of BP neural network model.

fragile critical point in 147 years.

\section{The Effect of Human Intervention and the Cost Prediction}

As the effect of climate change, the paper studies the impact of Brazil interventions on its fragility. Among a range of interventions taken in Brazil, indicators with more significant correlation to fragility are selected. The paper divides human interventions into four areas: control of carbon dioxide emissions, control of electricity consumption, the use of renewable energy for power generation and increasing forest area. Finally, the paper calculates the weight of the four human interventions on fragility and GDP and predicts the total cost.

\subsection{The Effect of Human Intervention}

First, the paper calculates the correlation coefficients of the interventions on climate change, which is shown in Table 5.

According to the comprehensive evaluating model, the weight of indexes on the state's fragility is analyzed and then revised continuously after adding human interventions. The results are shown in Table 6. Then, the weights of the interventions for fragility are given in Table 7.

Among national interventions, the weight of power generation from renewable sources on fragility is $60.028 \%$. It shows that renewable energy plays a decisive role in future climate change.

Finally, the paper calculates the fragility with and without human interventions, and compares to Figure 6. 
The effects of human intervention were analyzed. After calculating the percentage of fragility change, the combined impact of all interventions reduced fragility by $10.526 \%$.

Table 5. Correlation of interventions to climate change indicators.

\begin{tabular}{ccc}
\hline Interventions & Correlation with rainfall & Correlation with temperature \\
\hline Carbon dioxide emissions & -0.475 & 0.587 \\
Electricity consumption & -0.471 & 0.598 \\
Renewable energy for power generation & -0.422 & 0.683 \\
Forest area & 0.348 & -0.582 \\
\hline
\end{tabular}

Table 6. The weight of indexes on state's fragility.

\begin{tabular}{|c|c|c|c|}
\hline Fragile states index & Initial weight & First revised weight & The final revised weight \\
\hline Security Apparatus & 0.055665 & 0.055665 & 0.18909 \\
\hline Factionalized Elites & 0.079737 & 0.079737 & 0.016541 \\
\hline Group Grievance & 0.031414 & 0.031414 & 0.10938 \\
\hline Economy & 0.078476 & 0.078476 & -0.034065 \\
\hline Economic Inequality & 0.02222 & 0.02222 & 0.19156 \\
\hline Human Flight and Brain Drain & 0.06885 & 0.06885 & 0.19094 \\
\hline State Legitimacy & 0.044825 & 0.044825 & 0.16967 \\
\hline Public Services & 0.04389 & 0.04389 & 0.084149 \\
\hline Human Rights & 0.026119 & 0.026119 & -0.15455 \\
\hline Demographic Pressures & 0.03051 & 0.03051 & -0.1098 \\
\hline Refugees and IDPs & 0.03579 & 0.03579 & 0.068325 \\
\hline External Intervention & 0.0971 & 0.0971 & 0.27873 \\
\hline Rainfall & 0.033389 & -0.84753 & \\
\hline Temperature & 0.0045033 & 1.2329 & \\
\hline Carbon dioxide emissions & 0.080653 & & \\
\hline Electricity consumption & 0.051647 & & \\
\hline $\begin{array}{c}\text { Renewable energy for power } \\
\text { generation }\end{array}$ & 0.21023 & & \\
\hline Forest area & 0.0049752 & & \\
\hline
\end{tabular}

Table 7. The weights of the interventions for fragility.

\begin{tabular}{cc}
\hline Interventions & Weight \\
\hline Carbon dioxide emissions & 0.14977 \\
Electricity consumption & 0.23243 \\
Renewable energy for power generation & 0.60028 \\
Forest area & 0.01752 \\
\hline
\end{tabular}




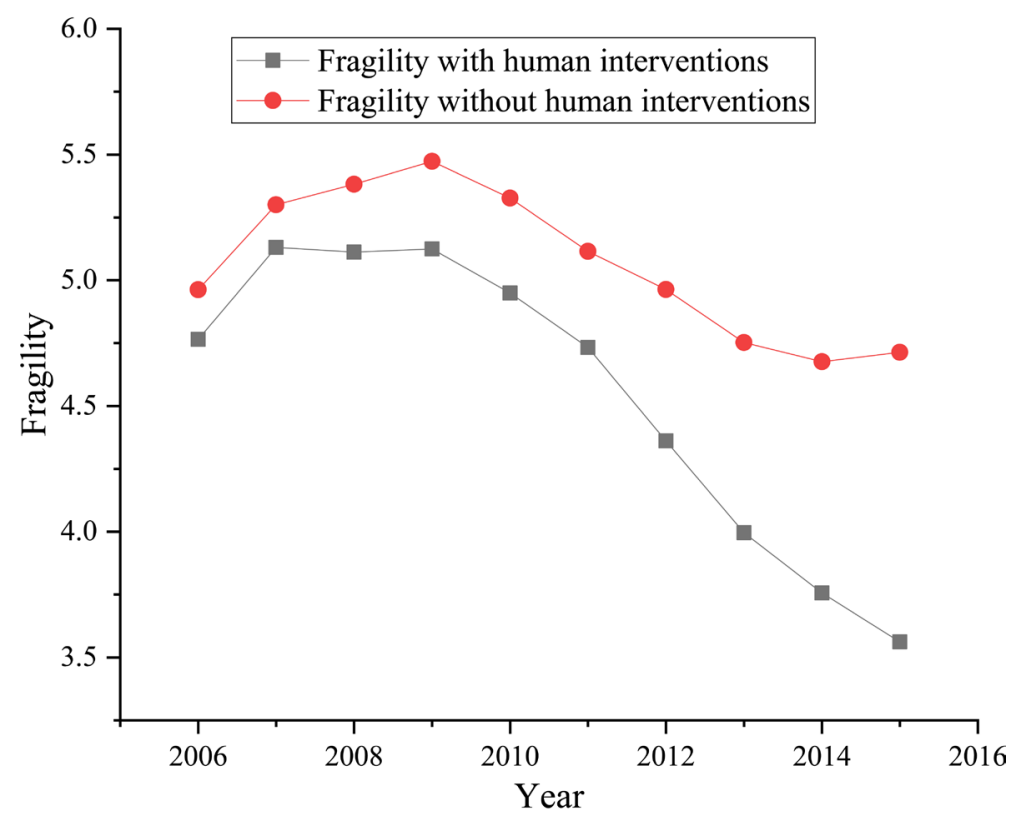

Figure 6. Fragility with and without human interventions.

Table 8. The weight of Intervention for GDP in Brazil.

\begin{tabular}{cl}
\hline Interventions & Weight \\
\hline Carbon dioxide emissions & 0.2499 \\
Electricity consumption & 0.2500 \\
Renewable energy for power generation & 0.2501 \\
Forest area & 0.2500 \\
\hline
\end{tabular}

\subsection{Predict the Total Cost of Interventions}

To analyze the total cost of interventions, the paper adds GDP as another factor and studies the weight of intervention for GDP in Brazil, which is shown in Table 8.

Based on the weight of interventions for fragility and GDP, the paper takes the data into the BP neural network prediction model. The result shows that the total national GDP in Brazil has reduced by $8.654 \%$. Simultaneously, fragility has decreased by $10.526 \%$, which means the total cost of interventions is $8.654 \%$ of Brazil's GDP.

\section{Conclusion}

This paper developed a comprehensive evaluating model to output the objective comprehensive evaluating results of state's fragility. Six representative countries were selected for validation and their predicted fragile value was consistent with the real situation. The state's fragility and the fragile tipping point were also predicted by BP neural network. This paper also analyzed the impacts of human interventions on improving state's fragility. The results indicated that the reasonable suggestions for the government were provided in the aspects of when and 
how to take what kind of interventions to improve state's fragility.

\section{Conflicts of Interest}

The authors declare no conflicts of interest regarding the publication of this paper.

\section{References}

[1] Adger, W.N. (1997) Social Vulnerability to Climate Change and Extremes in Coastal Vietnam. World Development, 27, 249-269.

https://doi.org/10.1016/S0305-750X(98)00136-3

[2] Yu, O. and Yan, J. (2011) Reviews on Regional Climate Change Vulnerability Assessment. Progress in Geography, 30, 27-34.

[3] He, X. (2018) Research on the Impact of Climate Change on the Fragility of a Country Based on Analytic Hierarchy Process. IOP Conference Series. Earth and Environmental Science, 208, Article ID: 012120. https://doi.org/10.1088/1755-1315/208/1/012120

[4] Peng, Z.D., Men, Z.Y. and Wu, X.F. (2019) Assessment and Prediction of the Fragility of a Country and the Influence of Climate Change. AIP Conference Proceeding, 2122, Article ID: 020004. https://doi.org/10.1063/1.5116443

[5] Chen, K.W. (2019) Model for Measuring Country's Fragility under the Influence of Climate Changes. IOP Conference Series: Earth and Environmental Science, 252, Article ID: 042008. https://doi.org/10.1088/1755-1315/252/4/042008

[6] Chen, Q., Liu, G., Liu, L., et al. (2019) The Evaluation System Model of Fragility to Counter THE Climate Change. IOP Conference Series. Earth and Environmental Science, 227, Article ID: 062006. https://doi.org/10.1088/1755-1315/227/6/062006

[7] Lu, M. and Kong, Y.F. (2014) Advances in Ecological Sensitivity Assessment. Journal of Shandong University of Architecture, 29, 347-352.

[8] Shi, P.J. (2016) Climate Change Risk and Its Comprehensive Prevention. Insurance Theory and Practice, No. 1, 69-85.

[9] Shi, P.J., Wang M., Hu, X.B. and Ye, T. (2014) Cohesion Mode of Social-Ecosystem Integrated Risk Prevention. Acta Geographica Sinica, 69, 863-876. 